\title{
Time ontology with Reference Event based Temporal Relations (RETR)
}

\author{
M.Hemalatha ${ }^{1}$, V. Uma ${ }^{2}$ and Dr. G. Aghila $^{3}$ \\ ${ }^{1}$ Department of Computer Science, Pondicherry University, India \\ hemalathamohanrajegmail.com \\ ${ }^{2}$ Assistant Professor, Department of Computer Science, Pondicherry University, India \\ umabskr@gmail.com \\ ${ }^{3}$ Professor, Department of Computer Science, Pondicherry University, India \\ aghilaa@yahoo.com
}

\begin{abstract}
:
Ontology of time is gaining importance as it is necessary to realize the Semantic Web. They play an important role in Natural Language Processing applications, data mining, decision support systems etc. Existing time ontologies have been represented using DAML, OWL, KSL, KIF and Cyc. In time ontology, events are concepts and topological temporal relations between events are either Point based or Interval based. Allen has proposed temporal interval relations which have been represented in the existing time ontologies. Among the Allen's 13 temporal relations the 'before' relation is ambiguous as it is context dependent. In this work, the 'before' relation has been augmented with 3 more specific relative event based temporal relations which increases the efficiency of temporal reasoning. A temporal ontology when created for medical domain using Protégé OWL can be an efficient decision support system when the additional temporal relations RETR are incorporated.
\end{abstract}

\section{KEY WORDS:}

Semantic web,ontology, time ontology, temporalrelations, referenceevent

\section{INTRODUCTION:}

The semantic web is a weave of information interlinked with each other in such a way that it can be easily understood by the machines and the information available can be easily handled globally on a large scale. So, it can also be represented efficiently as a globally linked database. Now the semantic web is playing a major role and the researchers are trying to incorporate this upcoming semantic web concept into the existing web. This semantic web is built using ontologies. Gruber [1] stated that ontology is the explicit specification of conceptualization. It describes the knowledge of a specific domain in an abstract and simplified view which can be further used for different purposes. The ontology can either be built afresh or can reuse the existing ontology. The domain can be explicitly explained with the help of ontology which involves concepts,objects, entities and relationships existing among them [1].Ontologies are used

DOI : 10.5121/ijwest.2012.3103 
International Journal of Web \& Semantic Technology (IJWesT) Vol.3, No.1, January 2012

in various fields like philosophy, artificial intelligence, database, softwareengineering, computer science $[2,3]$.The developers in these fields, uses ontology in order to make the machines to understand the domain, and to enable the semantic process by computers. The ontology helps both the system and the user to communicate with each other and helps in providing a common and shared understanding of the domain. These domains involve time as a major component. Representing this time in the ontology is the main aspect of any knowledge base since changes and actions are performed often. The temporal information is abundant in real world situations. The time ontology can also be depicted as a specification of a conceptualization for temporal knowledge. This describes temporal content of Web pages and the temporal properties of Web services. This OWL time is the next version of DAML time ontology. There are different ways of representing the time ontology [7] like instant based time theories and Allen's interval based time theory. Allen's interval algebra represents 13 basic temporal relations. The temporal relations are used to represent the directionality to time. These temporal relations are enabled to provide the ordering of events that had happened.Amongst the 13 relations provided by Allen, 'before' relation is context dependent. The ambiguity in 'before' relation can be reduced when augmented with additional reference event based temporal relations. The reference event is simply another event in the set of events provided. With the help of reference event, the computation can be provided. The ontology that uses the reference event for computation can be queried with the help of the temporal reasoning.

The paper is as follows: Section 2 deals with the related work done in time ontologies, Section 3 details Allen's temporal relations, Section 4 elaborates the proposed approach and Section 5 concludes the paper.

\section{RELATED WORKS}

Temporal information is available abundantly in web. Each and every service in the web includes the temporal information as a part of it. So in such a case, the web service should be able to provide the temporal information in a proper way. For this need, OWL-T has been developed, which supports the temporal properties of web service and the temporal content of web pages [5]. For any information in the web, time is the most important and the basic attribute. With this time, the temporal information processing has become an efficient process in the field of information system, semantic web and natural language processing [6]. This type of temporal ontologies provides computation of time and this type of ontologies play a major role in the natural language processing. Evident time ontologies have been built: DAML ontology of time, the time ontology in OWL, KSL time ontology, the time ontology in KIF, the temporal portion of IEEE Standard Upper Ontology.

These ontologies share a common understanding about their domain. The DAML ontology of time uses first-order predicate calculus axioms. These axioms can be focused to describe the other temporal theories that are more specific [7].The time ontology inOWL expresses the facts about topological relations among instants and intervals, together with information about durations, and about date time information [8]. Similar to the DAML ontology of time, this also supports the first order logic calculus. Both the DAML ontology and the time ontology in OWL, considers the instants and intervals as the temporal primitives. The KSL time ontology[Knowledge Systems Laboratory] is the simple time ontology that considers both the time points and time interval as primitive elements on a time line[4].This ontology contains a hierarchical structured class. It involves the relation between time interval and time points, axioms and time granularity. KSL 
International Journal of Web \& Semantic Technology (IJWesT) Vol.3, No.1, January 2012

time ontology differentiates the closed and open intervals. KIF ontology [Knowledge Interchange Format] is a language that is mainly proposed for exchanging the knowledge between different systems. These languages are mainly based on predicate logic semantically and LISP syntactically .The time ontology in KIF includes the first order predicate logic. Cyc's Upper Ontology [9] is contained in Cyc Knowledge Base. This models temporal points and time intervals. The key concept in this ontology is the temporal thing, which is described by the properties duration, ending point and ending date.

Before the advent of the Allen's proposal, there are different form of calculus evaluated in the field of logical formalism. This logical formalism is used in the ontological engineering. Kahn and Gory [10]provide a solution for the representation of time dependent knowledge. They considered time as a composition of points. The beginning and the end point of the interval are represented as non-punctual events. Time intervals are the distance between any two points. In situation calculus [11], series of situations are represented as knowledge. Each situation acts as a description of the world at particular point of time.If a particular fact in situation calculus is proved to be true, then it should be reprove as true in its succeeding instants of time [15].Actions and events maps state to state. The Event calculus uses the logical language for representing and reasoning about actions and their effects.

\section{ALLEN'S TEMPORAL RELATIONS}

Allen proposed a framework for temporal reasoning. All the relation proposed by Allen deals with the directionality to time. This reasoning includes the interval based temporal logic. Intervals are the only temporal primitives in this temporal logic [16]. Allen's main aim is to exemplify natural language sentences and to represent plans. Therefore Allen defined 13 basic relations between time intervals. In these basic intervals, six of the intervals are the inverse of the other six:

before, after, finishes, finished-by, overlaps, overlapped-by, starts, started- by, during, contains, meets, met-by and equal[15]. These temporal relations depict and relate the actions and plans. For easy access the basic temporal ontologies have been categorized into sub ontology with instants and intervals [12].Fig 1 represents the Allen's interval based temporal relations. 
International Journal of Web \& Semantic Technology (IJWesT) Vol.3, No.1, January 2012

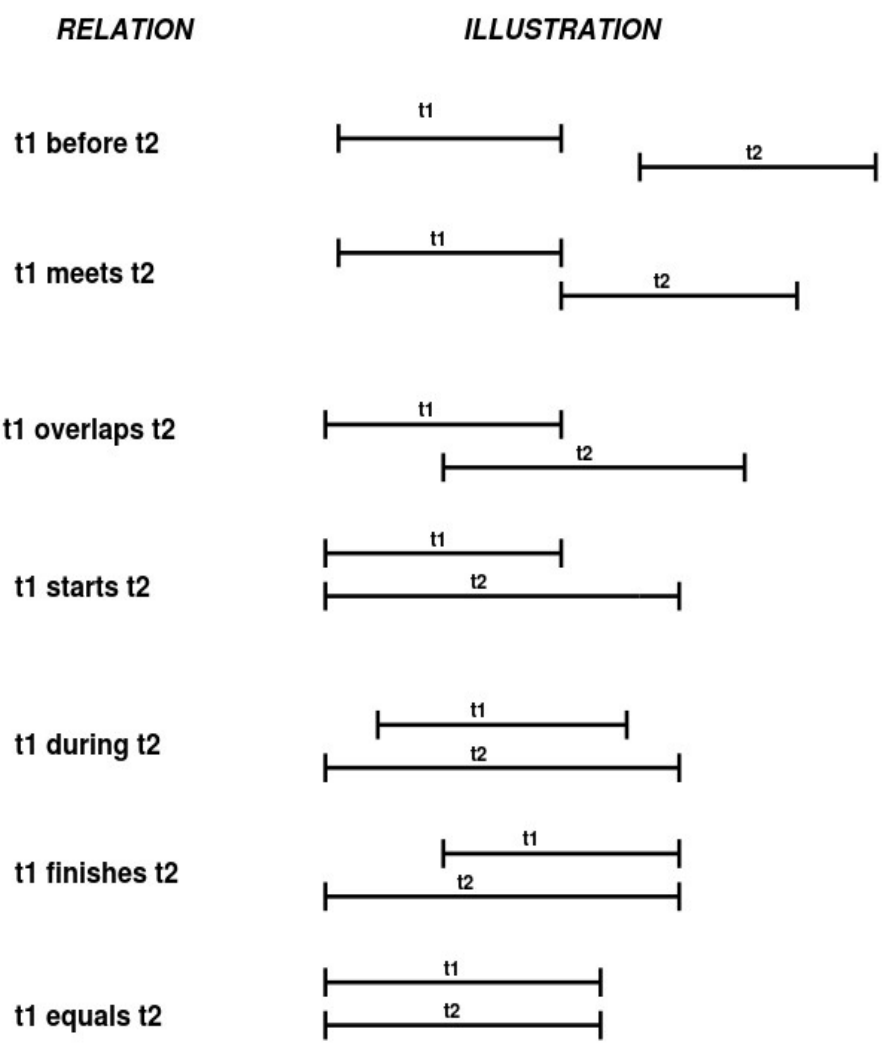

Fig 1: Allen notation

Time can refer either to points or intervals. The time point is any event at particular point of time.

Example

The exam started at 10 in the morning.

This example discuss the event at a particular point of time. Time can also refer to intervals where the act of finding the event can be within the interval provided.

Example

My friend wrote the exam from 2 to 5 in the afternoon.

In the above example the act of finding the event occurs within the interval "today" instead of providing a particular instant of time. Both the examples deal with the time representation. 
International Journal of Web \& Semantic Technology (IJWesT) Vol.3, No.1, January 2012

\section{PROPOSED APPPROACH:}

The semantic web is an upcoming technology mainly developed to enable users to find, share, and combine information more easily. This semantic web primarily uses ontology for representing the data within it. The temporal concepts are widely scattered in the web. This information is stored in the knowledge base which can be used when ever needed. Time ontologies are intended to give a specification of a conceptualization of temporal contents of web information and temporal properties of web services [13]. There are different types of time ontologies developed at various sites. As described in earlier section, evident time ontologies have been developed across various laboratories. These time ontologies uses Allen's representation for developing an ontology. Allen has proposed 13 relations for the events described in the ontology. These relations basically depict the occurrence of each event and how they are related with each other in time line. The temporal relation thus provides ordering of the events occurred.The "before "relation of the Allen is context dependent and it is always ambiguous.

\section{Example}

Events that happened yesterday are also considered as before and event that happened two days back is also considered to be before. There is no ordering of events in such a case.

With the existing approach, when a query with 'before' relation is given, then, it retrieves all the events described before that instant of time. For example, consider four events A, B, C, D happening at instants of time $\mathrm{t} 1, \mathrm{t} 2, \mathrm{t} 3, \mathrm{t} 4$ respectively. Now say a query, "event before $\mathrm{t} 4$ ", then it responds with the events before that specific time (i.e.) A, B, C will be retrieved.This relation can be enabled with the help of two functions: hasStart and hasEnd.hasStart is the start time of a particular event and hasEnd is the end time of the same event. Any event has start time and end time associated with it. Thus in the existing approach, in fig 2, the start time of the second event is compared with the end time of the first event. In this comparison, if the start time of the second event say B is after the end time of the first event say A, then it can be concluded that event A is before event $\mathrm{B}$. Thus, in the existing approach, the results obtained are with less precision where all the events before a particular time event is considered to be before that necessary time instant.

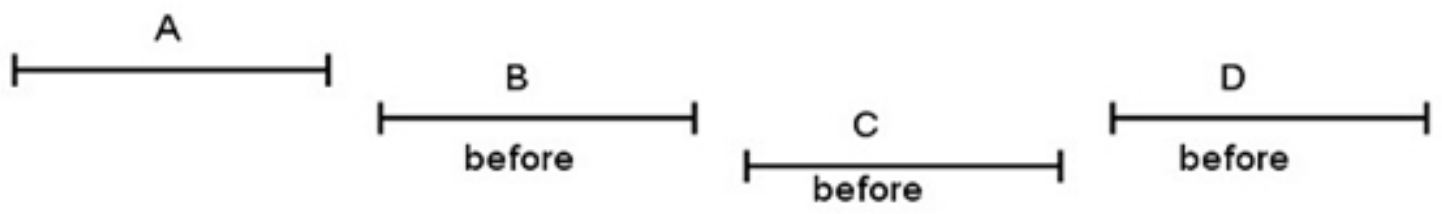

Fig 2: Ambiguity in 'before' relation

For any before relation,

1. $\forall(\mathrm{t} 1, \mathrm{t} 2)$ before $(\mathrm{t} 1, \mathrm{t} 2) \rightarrow \neg$ before $(\mathrm{t} 2, \mathrm{t} 1)$

2. $\forall(\mathrm{t} 1, \mathrm{t} 2, \mathrm{t} 3, \mathrm{t} 4)($ before $(\mathrm{t} 1, \mathrm{t} 2) \wedge$ before $(\mathrm{t} 2, \mathrm{t} 3) \wedge$ before $(\mathrm{t} 3, \mathrm{t} 4) \rightarrow$ before $(\mathrm{t} 1, \mathrm{t} 4)$ 
International Journal of Web \& Semantic Technology (IJWesT) Vol.3, No.1, January 2012

\section{3. $\forall \mathrm{T} \forall \mathrm{t} 1 \forall \mathrm{t} 2(($ hasStart $(\mathrm{T})=\mathrm{t} 1) \Lambda(\operatorname{hasEnd}(\mathrm{T})=\mathrm{t} 2) \rightarrow$ before $(\mathrm{t} 1, \mathrm{t} 2))$}

\section{Example}

In a medical ontology, the case history of a patient is represented using topological relations of of the symptoms of a disease and if a query is given to know the event(symptom) that has occurred before a particular symptom, then the system retrieves with more patients 'symptom without any categorization. Now, it becomes a tedious task for the person to identify the disease of a particular patient since the data set retrieved is huge and ordering is not clear. This can be overcome with the proposed method where a reference event is considered for categorizing the above events.

In fig3, an ontology is created in protégé with event as main class and games and disaster as sub class for the main event.Each of the class has two individuals(events) within it where each individual has a start time and end time associated with it. When a query is given with this start time and end time, the result is obtained as shown in fig4 showing the ambiguity in 'before' relation.A reasoner is used to evaluate the query.

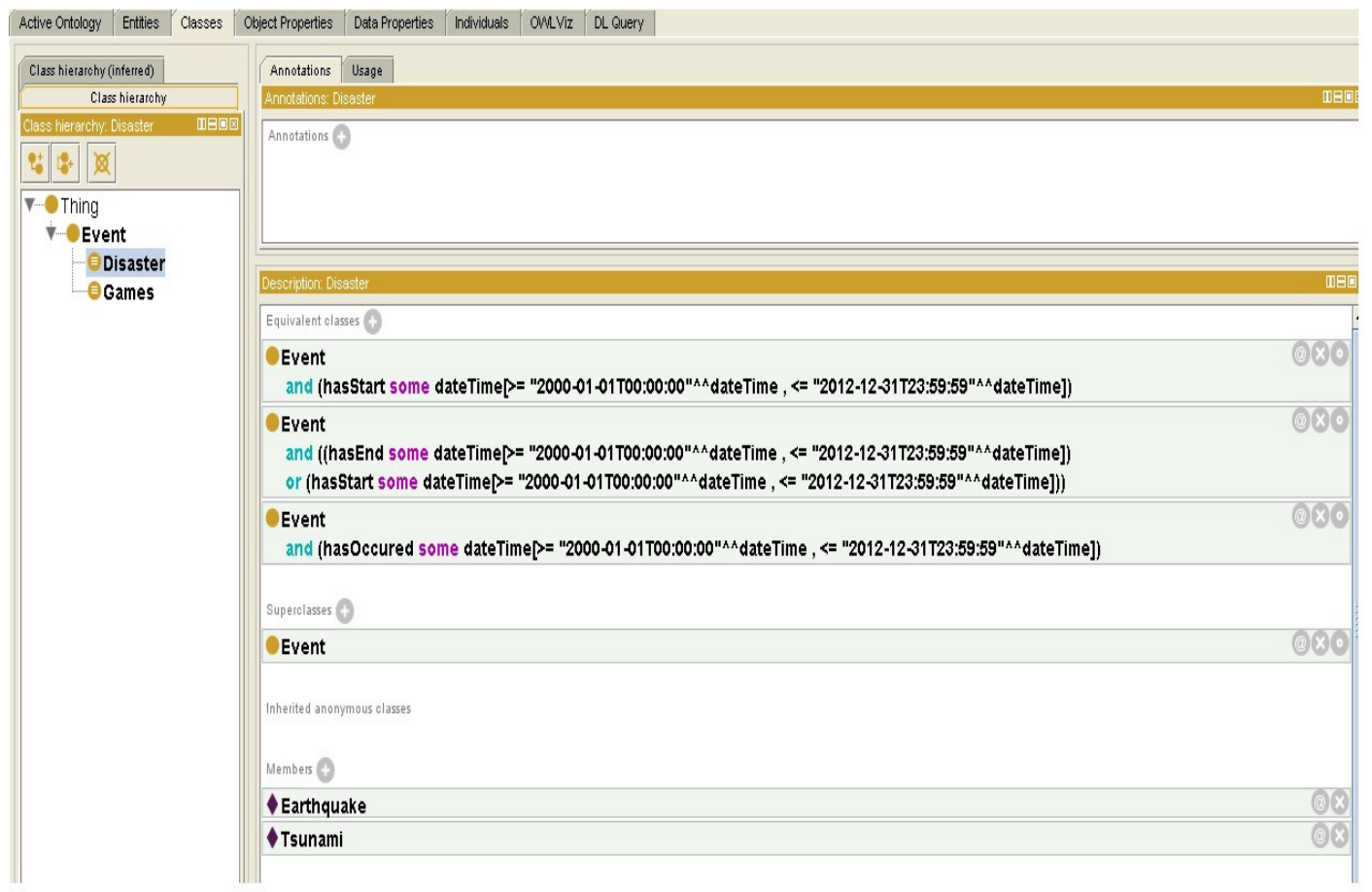

Fig 3 : Events with hasStart,hasEnd and has Occured properties 
International Journal of Web \& Semantic Technology (IJWesT) Vol.3, No.1, January 2012

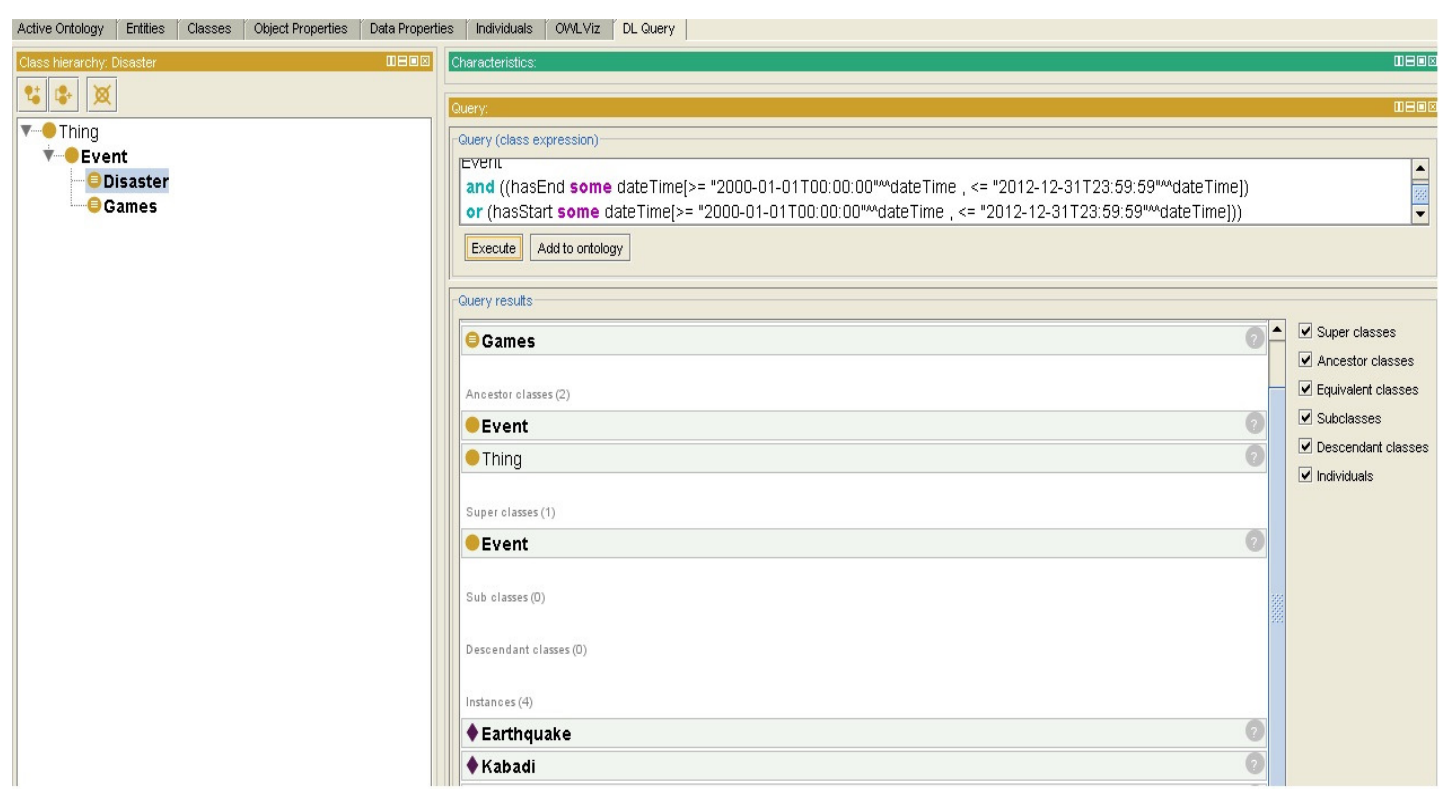

Fig 4: query with has Start and has End relation

So, the 'before' relation of Allen' is further sub-classified into 4 such sub-relations like 'shortly before', 'quite before', 'long before' and 'extremely before'. These categorizations are made with the help of reference event.The reference is event is also an attribute of time which has its own start and end time. Therefore the reference event is another event used in ontology. The mid value of the reference event is calculated by considering the start time and end time. Consider 4 events, say $\mathrm{A}, \mathrm{B}, \mathrm{C}$ and $\mathrm{D}$. If the mid value of the reference event is after the start time of the event $\mathrm{B}$, then that event is referred as shortly before (i.e.) event A is shortly before event B. In the fig 5, with the reference event $\mathrm{F}$, the event $\mathrm{A}$ shortly before the event $\mathrm{B}$, quite before event $\mathrm{C}$, long before event $\mathrm{D}$ and extremely before event $\mathrm{E}$. With such a categorization of event, the accurate information can be retrieved.

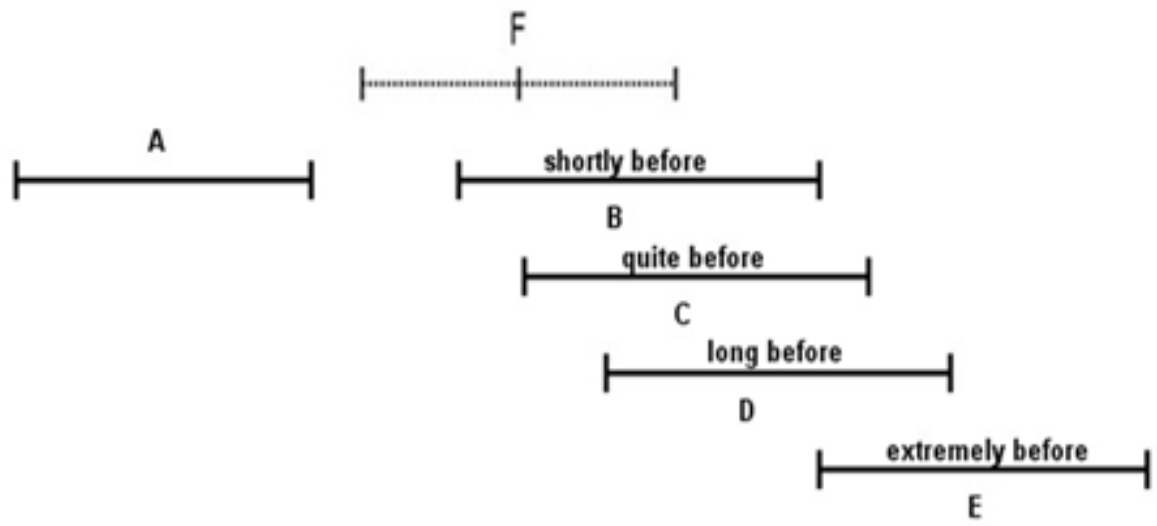

Fig 5: Reference event based temporal relation 
International Journal of Web \& Semantic Technology (IJWesT) Vol.3, No.1, January 2012

The time stamp of any event is compared with the time stamp of the reference event $(F)$, and the 'before' relation can be classified. With such a classification, the exact event can be obtained without much ambiguity.

The following axioms can be defined for the new RETR.

shortlybefore $(\mathrm{T} 1, \mathrm{~T} 2) \equiv(((\mathrm{endfn})(\mathrm{T} 1)$ before $\operatorname{startfn}(\mathrm{T} 3)) \mathrm{V}$ (endfn(T1) meets $\operatorname{startfn}(\mathrm{T} 3))) \wedge(\operatorname{midfn}(\mathrm{T} 3)$ after $\operatorname{startfn}(\mathrm{T} 2))$

quitebefore $(\mathrm{T} 1, \mathrm{~T} 2) \equiv(((\mathrm{endfn}$ (T1) before $\operatorname{startfn}(\mathrm{T} 3)) \mathrm{V}(\mathrm{endfn}(\mathrm{T} 1)$ meets $\operatorname{startfn}(\mathrm{T} 3))) \wedge$ (midfn(T3) equals startfn(T2))

longbefore $(\mathrm{T} 1, \mathrm{~T} 2) \equiv(((\mathrm{endfn}$ (T1) before $\operatorname{startfn}(\mathrm{T} 3)) \mathrm{V}(\mathrm{endfn}(\mathrm{T} 1)$ meets $\operatorname{startfn}(\mathrm{T} 3))) \wedge$ (midfn(T3) before startfn(T2))

extremelybefore $(\mathrm{T} 1, \mathrm{~T} 2) \equiv(((\mathrm{endfn}$ (T1) before $\operatorname{startfn}(\mathrm{T} 3)) \mathrm{V}(\mathrm{endfn}(\mathrm{T} 1)$ meets $\operatorname{startfn}(\mathrm{T} 3))) \wedge$ (endfn(T3) before startfn(T2))

where $\mathrm{T} 3$ is the reference event and the functions startfn, endfn, midfn returns the start, end, mid instants of $\mathrm{T}$.

According to our proposal, these comparisons are done with SWRL(Semantic Web Rule Language)[14]. SWRL is a plugin developed which has in-built libraries in it.These in-built functions are used to evaluate the query. Rules are framed in this SWRL which is used in evaluating the query. An ontology for medical domain is developed with different SWRL rules incorporated in it.The SWRL rules can be framed as

Antecedent(body) --->consequent (head)Example

ReferenceEvent (?r) $\wedge$ hasStart (?f,?t1) $\wedge$ hasEnd (?f,?t2) $\Lambda$ swrlb:add(midpointvalue,?t1,?t2) $\rightarrow$ hasMidPointValue (?f,?midpointvalue )

Reasoners like Pellet, Fact ++, SPARQL ,Hermit the in-built plugins of Protégé can be used to reason out the query. When a query is given, the reasoner is started, and the query is analyzed. On analysis of the query, the consistency can be obtained. In this proposed approach, each and every event is provided with a time stamp.

\section{CONCLUSION}

In this paper, time is considered as an important factor. A reference event is proposed in which the mid-point of the reference event is considered for the classification of the temporal relations. The temporal relations are used to provide the ordering of events occurred. The ontology can be built and consistency of any query can be checked. Temporal reasoners are used to evaluate the query given. The reasoner works and an accurate result can be obtained. The result will be much accurate when compared to the earlier systems proposed. Since the development of the proposed system is still in its development stage, an account of actual implementation cannot be provided in this paper. Thus, this proposal provides an enhancement in the retrieval of information in the time ontology. 
International Journal of Web \& Semantic Technology (IJWesT) Vol.3, No.1, January 2012

\section{REFERENCES:}

[1] T.RGruber, "Toward Principles for the Design of Ontologies used for Knowledge Sharing," International Journal Human-Computer Studies, Vol. 43, No. 5-6, pp 907-928,1993.

[2] C. Calero, F. Ruiz and M. Piattini, "Ontologies for Software Engineering and Software Technology", Calero .Ruiz .Piattini(Eds)Springer-Verlag Berlin Heidelberg, 2006.

[3] C. A Welty, "Ontology Research,” AI Magazine, Vol. 24, No. 3, pp 11-12, 2003.

[4] Qing Zhou, Richard Fikes: A Reusable Time Ontology-Proceeding of the AAAI Workshop on Ontologies for the Semantic Web,2002 .

[5] Tim BernesLee,JamesHendler andOraLassila," The Semantics Web: Scientific American”, 284(5) ,pp34-43,2001.

[6] Feng Pan and Jerry R.Hobbs , "Time Ontology in OWL”World Wide Web Consortium, Working Draft WD-owl-time-20060927, 2006.

[7] Jerry R. Hobbs , “ A DAML Ontology of Time “,2002.

[8] Feng Pan and Jerry R. Hobbs, "Time in OWL-S" Proceedings of the AAAI Spring Symposium on Semantic Web Services, , pp. 29-36, AAAI Press,2004

[9] Martin.O.'Connor, Ravi.D.Shankar, David B.Parish, AmarK.Das "Knowledge Data Integration For The Temporal Reasoning In A Clinical Trial System" International Journal Of Medical Informatics, 78 Suppl 1,S77-85,2009.

[10] Carlo Combi,ElpidaKeravnore-Papailiou,YuvalShahar,'Temporal Information In Medicine",Springer ,pp 113, 2010.

[11] FengPan,"An Ontology of Time: Representing Complex Temporal Phenomena for the Semantic Web and Natural Language", ACM digital library, 2007.

[12] ElzbietaHajnicz,"Time Structures:Formal Description and Algorithmic Representation”, SpringerVerlag Berlin Heidelberg, pp233, 1996

[13] Laure Vieu,Achille C. Varzi , "Formal Ontology In Information System"Proceedings Of The Third Conference(FOIS-2004), 2004.

[14] Jerry R. Hobbs and Feng Pan "Time Ontology in OWL". Ontology Engineering Patterns Task Force of the Semantic Web Best Practices and Deployment Working Group, World Wide Web Consortium (W3C) notes,2006.

[15] James. F. Allen, "Maintaining Knowledge about Temporal Intervals "Communications of ACM ,Vol 26 , no 11 ,November 1983

[16] Mostafa E. Saleh , "Semantic-Based Query in Relational Database Using Ontology"Journal on Data, Information and Knowledge Engineering,Vol. 2, No. 1, January 2011. 